\title{
PACIENTES SUBMETIDOS A CIRURGIAS BARIÁTRICAS: FATORES ASSOCIADOS A COMPLICAÇÕES PÓS-OPERATÓRIAS DE SÍTIO CIRÚRGICO
}

\author{
Patients undergoing bariatric surgeries: factors associated \\ with post-operative complications from the surgical site \\ Pacientes sometidos a cirugías bariátricas: factores \\ asociados con complicaciones postoperatorias del sitio quirúrgico \\ Priscilla Vasconcelos Aguiar ${ }^{1}$, Eduardo Tavares Gomes ${ }^{2 *}$, \\ Iraneide Nascimento dos Santos ${ }^{3}$, Aracele Tenório de Almeida e Cavalcanti ${ }^{4}$
}

RESUMO: Objetivo: Verificar a prevalência e os fatores associados às complicações pós-operatórias de sítio cirúrgico em pacientes submetidos a cirurgias bariátricas. Método: Estudo transversal, retrospectivo, analítico, com abordagem quantitativa. Foram analisados 197 casos de pacientes obesos submetidos à cirurgia bariátrica entre janeiro de 2013 e janeiro de 2016 em Pernambuco, Brasil. As variáveis relacionadas dicotomizadas foram analisadas por teste do $\chi^{2}$. O risco de complicações foi estimado pela odds ratio (OR). Assumiu-se significância de $\mathrm{p}<0,05$. Resultados: Entre os 30 pacientes que compuseram a amostra, foram observadas 45 complicações pós-operatórias de sítio cirúrgico. Houve maior incidência nos indivíduos acima de 45 anos (70,0\%). Dos fatores que poderiam estar associados aos desfechos, destacaram-se abordagem cirúrgica aberta $(\mathrm{OR}=5,35)$, inserção de drenos $(\mathrm{OR}=4,48)$ e período de tempo de pós-operatório superior a 3 dias de internação $(\mathrm{OR}=5,03)$. Conclusão: Comprovou-se maior prevalência de seroma como complicação de sítio cirúrgico, além da técnica cirúrgica (bypass em Y de Roux), faixa etária maior de 45 anos, tipo de abordagem cirúrgica convencional/aberta, presença de inserção de dreno cavitário e tempo de internação superior a 3 dias como predisponentes a um maior desenvolvimento de complicações.

Palavras-chave: Complicações pós-operatórias. Cirurgia bariátrica. Obesidade mórbida.

ABSTRACT: Objective: To verify the prevalence and the factors associated with postoperative surgical site complications in patients undergoing bariatric surgeries. Method: A cross-sectional, retrospective, analytical study with a quantitative approach. 197 cases of obese patients undergoing bariatric surgery were analyzed between January 2013 and January 2016 in Pernambuco, Brazil. Dichotomized variables were analyzed using the $\chi^{2}$ test. The risk of complications was estimated by the odds ratio (OR). A significance of $\mathrm{p}<0.05$ was assumed. Results: Among the 30 patients that made up the sample, 45 postoperative surgical site complications were observed. There was a higher incidence in individuals over 45 years of age ( $70.0 \%)$. The factors that stood out as possibly being associated with the outcomes were: an open surgical approach (OR $=5.35)$, the insertion of drains $(\mathrm{OR}=4.48)$, and a postoperative period longer than 3 days of hospitalization $(\mathrm{OR}=5.03)$. Conclusion: The following showed a high disposition for the development of complications from the surgical site: a high prevalence of seroma, the Roux-en-Y bypass surgical technique, the patient's age over 45 years old, a conventional/ open surgical approach, the insertion of cavitary drainage, and a hospitalization stay longer than 3 days.

Keywords: Postoperative complications. Bariatric surgery. Morbid obesity.

'Enfermeira; mestranda em Neuropsiquiatria e Ciências do Comportamento pela Universidade Federal de Pernambuco (UFPE); especialista em Enfermagem Cirúrgica, modalidade Residência em Enfermagem, pela Universidade Federal de Pernambuco (UFPE) - Recife (PE), Brasil.

¿Enfermeiro assistencial da Unidade de Centro Cirúrgico do Hospital das Clínicas (HC) da UFPE; mestre em Enfermagem pelo Programa Associado de Pós-Graduação em Enfermagem (PAPGEnf) da Universidade de Pernambuco (UPE)/Universidade Estadual da Paraíba (UEPB); especialista em Enfermagem em Suporte Avançado de Vida pela Faculdade de Enfermagem Nossa Senhora das Graças da Universidade de Pernambuco (FENSG/UPE); especialista em Enfermagem em Cardiologia pelo Pronto-Socorro Cardiológico de Pernambuco (PROCAPE) da Secretaria Estadual da Saúde de Pernambuco (SES-PE) - Olinda (PE), Brasil. ${ }^{3}$ Enfermeira; mestre em Patologia; especialista em Enfermagem do Trabalho pela UFPE - Recife (PE), Brasil.

${ }^{4}$ Doutoranda e mestre em Ciências da Saúde pela Faculdade de Ciências Médicas da UPE; especialista em Doenças Infecciosas e Parasitárias pela UPE; coordenadora do Programa de Residência de Enfermagem do HC da UFPE - Recife (PE), Brasil.

*Autor correspondente: edutgs@hotmail.com

Recebido: 02/11/2017 - Aprovado: 17/12/2017

DOI: $10.5327 / Z 1414-4425201800010006$ 
RESUMEN: Objetivo: Verificar la prevalencia y los factores asociados con las complicaciones del sitio quirúrgico postoperatorio em pacientes sometidos a cirugías bariátricas. Método: Estudio transversal, retrospectivo y analítico con enfoque cuantitativo. Se analizaron 197 casos de pacientes obesos sometidos a cirugía bariátrica entre enero de 2013 y enero de 2016, em Pernambuco, Brasil. Las variables dicotomizadas se analizaron usando la prueba $\chi^{2}$. Le riesgo de complicaciones es estimó mediante la odds ratio (OR). Se asumió una significancia de p $<0,05$. Resultados: entre los 30 pacientes em la muestra, 45 complicaciones postoperatorias fueran observadas en el sitio quirúrgico. Hubo una mayor incidencia em individuos mayores de 45 años ( $70,0 \%$ ). Los factores que se destacaron como posiblemente asociados con los resultados fueron: un abordaje quirúrgico abierto (OR = 5,35), la inserción de drenajes $(\mathrm{OR}=4,48)$, y un periodo postoperatorio mayor a 3 días de hospitalización $(\mathrm{OR}=5,03)$. Conclusión: Los siguientes mostraron una alta disposición para el desarrollo de complicaciones del sitio quirúrgico: alta prevalencia de seroma, técnica quirúrgica de derivación Roux-em-Y, edad del paciente mayor de 45 años, abordaje quirúrgico convencional/abierto, inserción de denaje cavitario y hospitalización por más de 3 días.

Palablas clave: Complicaciones posoperatorias. Cirurgía bariátrca. Obesidad mórbida.

\section{INTRODUÇÃO}

A intervenção cirúrgica é apenas parte do tratamento integral para a obesidade, que é prioritariamente baseado na promoção da saúde e no cuidado clínico longitudinal. Os custos envolvidos no cuidado em saúde são significativamente maiores para os pacientes que tratam a obesidade com técnicas cirúrgicas do que para aqueles que a tratam com técnicas não invasivas, sendo, portanto, imprescindível a definição de critérios seguros de indicação, a certeza do insucesso de outras abordagens e a reflexão constante sobre as complicações potenciais ${ }^{1,2}$.

A cirurgia bariátrica é indicada a indivíduos que apresentem: índice de massa corporal (IMC) $>50 \mathrm{~kg} / \mathrm{m}^{2}$; IMC $>40 \mathrm{~kg} / \mathrm{m}^{2}$ com ou sem comorbidades, sem sucesso do tratamento clínico longitudinal por, no mínimo, 2 anos, e que tenham seguido o protocolo clínico; ou IMC $>35 \mathrm{~kg} / \mathrm{m}^{2}$, com comorbidades como diabetes mellitus (DM) e/ou hipertensão arterial (HAS), risco cardiovascular, apneia do sono, entre outros ${ }^{3}$.

A ocorrência de complicações pós-operatórias, em geral, está relacionada a diversos fatores, entre eles: patologia clínica associada, tipo de anestesia, estágio do agravo que levou à cirurgia e cuidados pós-operatórios. Certos pacientes apresentam risco maior de apresentar complicações devido ao seu estado clínico pré-operatório, como é possível perceber nos indivíduos obesos ${ }^{4}$.

As complicações de sítio cirúrgico são importantes causas de morbidade pós-operatória imediata e tardia, principalmente pós-laparotomia. A cicatrização das feridas cirúrgicas em indivíduos normais e saudáveis ocorre por meio de uma sequência ordenada de eventos fisiológicos, que incluem inflamação, epitelização, fibroplasia e maturação. A falha mecânica ou falha de cicatrização da ferida no local da cirurgia pode levar à ruptura do fecho, ocasionando seroma, hematoma, deiscência da ferida ou hérnia. Outras complicações incluem infecção de sítio cirúrgico (ISC), hemorragias e isquemias 5 . Dessa maneira, a obesidade pode dificultar a cicatrização da incisão cirúrgica, estando associada ao aumento da incidência de ISC, formação de hematoma, hérnia incisional e complicações em geral ${ }^{6}$.

O surgimento das fístulas pós-operatórias também representa uma grave complicação que pode ocorrer nos pacientes submetidos ao tratamento operatório da obesidade. O aumento da pressão intraluminal causado por estenose distal, a tensão excessiva na linha de sutura, a isquemia tecidual e o hematoma são os seus fatores predisponentes ${ }^{7}$.

Hematomas e seromas são coleções de sangue e soro, respectivamente. São as complicações de sítio cirúrgico mais comuns e geralmente resultam da falha da hemostasia primária ou de uma diátese hemorrágica (por exemplo, anticoagulação), podendo causar abertura da ferida cirúrgica e predispor à infecção, uma vez que esse quadro permite às bactérias ter acesso às camadas mais profundas da pele e se multiplicar no fluido estagnado 8 .

Sendo assim, o reconhecimento das características definidoras de complicações particulares do paciente obeso relacionadas às complicações pós-cirúrgicas trará diversos benefícios à prestação dos cuidados, pois irá direcionar os enfermeiros, parte integrante da equipe multidisciplinar, à investigação, ao diagnóstico de condições clínicas e ao controle de riscos, indicando a possibilidade de unir organização, padronização de linguagem científica e qualificação da prática de enfermagem.

\section{OBJETIVO}

Verificar a prevalência e os fatores associados às complicações pós-operatórias de sítio cirúrgico em pacientes submetidos a cirurgias bariátricas. 


\section{MÉTODO}

Trata-se de um estudo transversal, retrospectivo, de caráter analítico, com abordagem quantitativa, realizado no Hospital das Clínicas (HC) da Universidade Federal de Pernambuco (UFPE). O hospital é referência no estado em cirurgia bariátrica, recebendo pacientes em alto grau de obesidade desde que iniciou suas atividades nessa área, em 1997. O hospital conta com dez salas para cirurgias de grande porte, não havendo sala exclusiva para cirurgia digestiva.

A amostra do estudo foi censitária, composta por todos os pacientes obesos submetidos a cirurgias bariátricas vinculadas ao HC entre janeiro de 2013 e janeiro de 2016. No período, foram realizadas 220 cirurgias, sendo incluídos no estudo 197 prontuários, uma vez que os demais não estavam disponíveis no Serviço de Arquivo Médico (SAME).

Inicialmente, foram identificados, por meio de busca ativa no ambulatório, os nomes e registros dos pacientes submetidos a gastroplastias, para, em seguida, proceder-se à coleta com resgate de prontuários no SAME.

O instrumento de coleta foi um formulário composto por checklist, referente aos aspectos sociodemográficos, clínicos e cirúrgicos da amostra em estudo. As complicações foram consideradas quando surgiram até 30 dias após a cirurgia.

O IMC foi o indicador utilizado para estimativa de gordura associada à composição corporal, por ser a medida mais utilizada no mundo para classificar indivíduos com problemas de obesidade $^{8}$. No estudo, os valores de obesidade foram considerados de acordo com o Ministério da Saúde (MS), que a define como IMC igual ou maior que $30,0 \mathrm{~kg} / \mathrm{m}^{2}$, sendo também subdividida em termos de severidade, relacionado à associação de outras morbidades. Assim: IMC entre 30,0 e 34,9 kg/ $\mathrm{m}^{2}$ indica obesidade grau I; IMC entre 35,0 e $39,9 \mathrm{~kg} / \mathrm{m}^{2}$, obesidade grau II; e IMC entre 40,0 e $44,9 \mathrm{~kg} / \mathrm{m}^{2}$, obesidade grau III ${ }^{9}$. Por conseguinte, indivíduos com IMC $>45,0 \mathrm{~kg} / \mathrm{m}^{2}$ são considerados superobesos ${ }^{3}$.

O software utilizado para confecção do banco de dados e análises estatísticas foi o programa Statistical Package for Social Sciences (SPSS) for Windows, versão 17.0.

Realizou-se análise estatística descritiva, calculando-se frequência simples e coletiva, média, desvio padrão e mediana. A comparação das proporções das complicações em função de variáveis relacionadas dicotomizadas foi realizada pelo teste do $\chi^{2}$. O risco de complicações foi estimado pela odds ratio (OR), apresentada com intervalo de confiança de 95\% (IC95\%). Assumiu-se nível de significância para resultados com valor de $\mathrm{p}<0,05$.
O projeto foi aprovado pelo Comitê de Ética e Pesquisa da Universidade Federal de Pernambuco, por meio do CAAE 52533116.4.0000.5208.

\section{RESULTADOS}

A amostra avaliada no estudo totalizou 197 pacientes submetidos às cirurgias bariátricas. O sexo feminino foi o mais prevalente, com $77,2 \%$ (152 pacientes). A faixa etária de até 45 anos foi a mais operada, com $82,2 \%$ (162 pacientes), em comparação com os pacientes com mais de 45 anos ( $n=35 ; 17,8 \%)$.

Dos pacientes que realizaram a cirurgia no período analisado, 59,9\% ( $\mathrm{n}=118)$ apresentavam HAS e 25,4\% ( $\mathrm{n}=50)$, DM. Além dessas, diversas comorbidades foram observadas, como dislipidemia, em 11,7\% $(\mathrm{n}=23)$ dos indivíduos, doença renal crônica, em 1,5\% ( $\mathrm{n}=3)$ deles, e outras, em 10,7\% $(\mathrm{n}=21)$. As comorbidades apresentadas poderiam ser mais de uma. Em relação à classificação do IMC, 44,2\% ( $\mathrm{n}=87)$ dos pacientes eram classificados como superobesos e 38,0\% $(\mathrm{n}=75)$ apresentavam obesidade grau III. Entre os demais, $15,7 \%(\mathrm{n}=31)$ possuíam obesidade grau II e 2,0\% ( $n=4)$, obesidade grau I. A média de IMC foi de $45,3 \mathrm{~kg} / \mathrm{m}^{2}$, o que caracteriza graus elevados de obesidade.

De acordo com as técnicas cirúrgicas utilizadas, 51,3\% $(n=101)$ realizaram o bypass em $Y$ de Roux e 48,7\% ( $n=96)$, o sleeve. Foi realizada a abordagem videolaparoscópica em $84,3 \%(\mathrm{n}=166)$ dos procedimentos. Além disso, dos 197 pacientes analisados, 20,3\% $(\mathrm{n}=40)$ apresentavam relatos de inserção de drenos cavitários no intraoperatório. Desses, 75,0\% $(\mathrm{n}=30)$ utilizaram drenos por sucção e $25,0 \%(\mathrm{n}=10)$ a drenagem ativa, aberta. A média de internação pré-operatória foi de 4,7 dias $( \pm 15,462)$ e a média de dias de permanência no pós-operatório foi de 4,2 dias $( \pm 6,591)$. Permaneceram internados por até 3 dias na enfermaria $58,9 \%(n=116)$ dos pacientes e $41,1 \%(n=81)$ deles continuaram internos por mais de 3 dias na unidade. Os dados estão descritos na Tabela 1.

Para a estimativa das complicações, foram registradas 2 complicações para 6 pacientes e 3 complicações para 1 deles, totalizando 45 registros de complicações. As principais complicações de sítio cirúrgico evidenciadas no estudo foram: hematoma em $8,9 \%(n=4)$, seroma em $31,1 \%(n=14)$, hemorragia em $4,4 \%$ $(n=2)$, isquemia em $2,2 \%(n=1)$, infecção em $6,7 \%(n=3)$, hérnia incisional em $15,5 \%(\mathrm{n}=7)$, fístula em $6,7 \%(\mathrm{n}=3)$, deiscência superficial em $11,1 \%(n=5)$, deiscência profunda em $11,1 \%(n=5)$ e lesão de pele em $2,2 \%(n=1)$, com um total de $45(15,2 \%)$ complicações pós-operatórias de sítio cirúrgico. Os três casos de infecção foram superficiais. A Tabela 2 descreve essas complicações. 
Na Tabela 3, apresenta-se a análise das variáveis dicotômicas pela OR para complicações pós-operatórias de sítio cirúrgico. Para esse cálculo, foi considerado o número de indivíduos que apresentaram complicações (30 pacientes) e não as

Tabela 1. Características dos pacientes submetidos à cirurgia bariátrica no Hospital das Clínicas da Universidade Federam de Pernambuco, 2013-2016.

\begin{tabular}{|c|c|c|}
\hline Variáveis & $\mathbf{n}$ & $\%$ \\
\hline \multicolumn{3}{|l|}{ Sexo } \\
\hline Feminino & 152 & 77,2 \\
\hline Masculino & 045 & 22,8 \\
\hline \multicolumn{3}{|l|}{ Faixa etária (anos) } \\
\hline Até 45 & 162 & 82,2 \\
\hline Mais de 45 & 035 & 17,8 \\
\hline \multicolumn{3}{|l|}{ Comorbidades } \\
\hline Diabetes & 050 & 25,4 \\
\hline Hipertensão & 118 & 59,9 \\
\hline Dislipidemia & 023 & 11,7 \\
\hline Doença renal crônica & 003 & 1,5 \\
\hline Outros & 021 & 10,7 \\
\hline \multicolumn{3}{|l|}{ Índice de massa corporal } \\
\hline 30 a $34,9 \mathrm{~kg} / \mathrm{m}^{2}$ (grau l) & 004 & 2,0 \\
\hline 35 a 39,9 kg/m² (grau II) & 031 & 15,7 \\
\hline 40 a $44,9 \mathrm{~kg} / \mathrm{m}^{2}$ (grau III) & 075 & 38,0 \\
\hline$\geq 45 \mathrm{~kg} / \mathrm{m}^{2}$ (superobeso) & 087 & 44,2 \\
\hline \multicolumn{3}{|l|}{ Técnica cirúrgica } \\
\hline Bypass em Y de Roux & 101 & 51,3 \\
\hline Sleeve & 096 & 48,7 \\
\hline \multicolumn{3}{|l|}{ Abordagem } \\
\hline Videolaparoscópica & 166 & 84,3 \\
\hline Aberta & 031 & 15,7 \\
\hline \multicolumn{3}{|l|}{ Tempo cirúrgico (horas) } \\
\hline Até 3 & 087 & 44,2 \\
\hline Mais de 3 & 110 & 55,8 \\
\hline $\begin{array}{l}\text { Uso de antibiótico } \\
\text { profilático }\end{array}$ & 196 & 99,5 \\
\hline Inserção de drenos & 040 & 20,3 \\
\hline Reabordagem cirúrgica & 008 & 4,1 \\
\hline $\begin{array}{l}\text { Internação na unidade de } \\
\text { terapia intensiva }\end{array}$ & 030 & 15,2 \\
\hline \multicolumn{3}{|c|}{ Pós-operatório em enfermaria (dias) } \\
\hline Até 3 & 116 & 58,9 \\
\hline Mais de 3 & 081 & 41,1 \\
\hline
\end{tabular}

complicações individualmente (45 complicações), visto que um mesmo paciente poderia apresentar mais de uma complicação.

Destaca-se que os pacientes que mais apresentaram complicações possuíam mais de 45 anos ( $\mathrm{p}=0,057 ; \mathrm{OR}=2,340$ ) e a técnica cirúrgica com maior evidência de complicações foi o bypass em $Y$ de Roux ( $\mathrm{p}=0,0067 ; \mathrm{OR}=1,9000$ ). Além disso, as variáveis que apresentaram um valor de significância de $\mathrm{p}<0,05$ foram: abordagem cirúrgica, sendo a abordagem aberta com maior ocorrência de complicações de sítio cirúrgico ( $\mathrm{p}<0,001$; $\mathrm{OR}=5,350)$, inserção de drenos $(\mathrm{p}<0,001 ; \mathrm{OR}=4,480)$ e período de tempo de pós-operatório ( $\mathrm{p}<0,001 ; \mathrm{OR}=5,030$ ). Não se observou significância para associação entre o número de dias internado no período pré-operatório e a ocorrência do desfecho.

\section{DISCUSSÃO}

Ao se analisar as ocorrências de complicações de sítio cirúrgico, $15,2 \%$ dos pacientes que realizaram o procedimento apresentaram algum tipo de complicação, sendo o seroma, a mais comum (31,1\%). Uma revisão sistemática incluindo estudos realizados na última década apresentou uma taxa de complicações de $17,0 \%{ }^{9}$.

A faixa etária até 45 anos foi a mais operada $(82,2 \%$ das cirurgias) em comparação com a de mais de 45 anos (17,8\%). Porém, ao se analisar a associação entre idade e presença de complicações, constatou-se que os pacientes com mais de 45 anos apresentaram um número maior de complicações pós -operatórias de sítio cirúrgico, cuja análise estatística destaca valor muito próximo à significância $(\mathrm{p}=0,057 ; \mathrm{OR}=2,340)$.

Tabela 2. Complicações de sítio cirúrgico entre os pacientes submetidos à cirurgia bariátrica no Hospital das Clínicas da Universidade Federal de Pernambuco, 2013-2016.

\begin{tabular}{|l|c|c|}
\hline Complicações* & $\mathbf{n}$ & $\%$ \\
\hline Seroma & 14 & 31,1 \\
\hline Hérnia incisional & 07 & 15,6 \\
\hline Deiscência superficial & 05 & 11,1 \\
\hline Deiscência profunda & 05 & 11,1 \\
\hline Hematoma & 04 & 8,9 \\
\hline Infecção & 03 & 6,7 \\
\hline Fístula & 03 & 6,7 \\
\hline Hemorragia & 02 & 4,4 \\
\hline Isquemia & 01 & 2,2 \\
\hline Lesões de pele & 01 & 2,2 \\
\hline Total & 45 & 15,2 \\
\hline
\end{tabular}

*Um mesmo paciente pode ter apresentado mais de uma complicação. 
Estudos mostram que o tratamento cirúrgico da obesidade em indivíduos com mais de 45 anos continua controverso. Embora nas diretrizes do Sistema Único de Saúde (SUS) a idade não seja considerada um fator limitante, para essa faixa etária prevalece o conceito de que deve ser avaliada individualmente a relação risco-benefício do procedimento ${ }^{10,11}$.

Além disso, o risco de comorbidades está relacionado com o IMC e com idade avançada, sendo que quanto mais elevados, maiores os riscos independentes para doenças associadas ao sobrepeso e à obesidade, além de maiores riscos de complicações pós-operatórias ${ }^{12}$. Foi constatado na pesquisa o elevado número de comorbidades, como HAS $(59,9 \%)$ e DM $(25,4 \%)$. Há evidência de que as complicações cirúrgicas não aumentam de forma independente da idade, sendo um procedimento seguro para maiores de $60 \operatorname{anos}^{13}$. Ou seja, com a idade, podem estar mais presentes as comorbidades

Tabela 3. Odds ratio para complicações de sítio cirúrgico em função de variáveis dicotômicas dos pacientes submetidos à cirurgia bariátrica no Hospital das Clínicas da Universidade Federal de Pernambuco, 2013-2016.

\begin{tabular}{|c|c|c|c|c|}
\hline \multirow{2}{*}{ Variáveis } & Sem complicações & Com complicações & \multirow{2}{*}{ OR (IC95\%) } & \multirow{2}{*}{ Valor $\mathbf{p}$} \\
\hline & $n(\%)$ & $n(\%)$ & & \\
\hline \multicolumn{5}{|l|}{ Sexo } \\
\hline Feminino & $131(86,20)$ & $21(13,80)$ & \multirow{2}{*}{$1,56(0,66-3,69)$} & \multirow{2}{*}{0,310} \\
\hline Masculino & $36(80,00)$ & $9(20,00)$ & & \\
\hline \multicolumn{5}{|l|}{ Faixa etária (anos) } \\
\hline Até 45 & $141(87,00)$ & $21(12,90)$ & \multirow{2}{*}{$2,34(0,96-5,64)$} & \multirow{2}{*}{0,057} \\
\hline Mais de 45 & $26(74,30)$ & $9(25,70)$ & & \\
\hline \multicolumn{5}{|l|}{ Diabetes mellitus } \\
\hline Diabéticos & $40(80,00)$ & $10(20,00)$ & \multirow{2}{*}{$1,58(0,69-3,67)$} & \multirow{2}{*}{0,277} \\
\hline Não diabéticos & $127(86,39)$ & $20(13,61)$ & & \\
\hline \multicolumn{5}{|l|}{ Hipertensão } \\
\hline Hipertensos & $70(88,61)$ & $9(11,39)$ & \multirow{2}{*}{$1,68(0,73-3,89)$} & \multirow{2}{*}{0,221} \\
\hline Não hipertensos & $97(82,20)$ & $21(17,80)$ & & \\
\hline \multicolumn{5}{|l|}{ Técnica cirúrgica } \\
\hline Bypass em Y de Roux & $81(80,20)$ & $20(19,80)$ & \multirow{2}{*}{$1,90(0,94-3,85)$} & \multirow{2}{*}{0,067} \\
\hline Sleeve & $86(89,60)$ & $10(10,40)$ & & \\
\hline \multicolumn{5}{|l|}{ Abordagem } \\
\hline Videolaparoscópica & $151(90,90)$ & $15(9,00)$ & \multirow{2}{*}{$5,35(2,92-9,79)$} & \multirow{2}{*}{$<0,001$} \\
\hline Aberta & $16(51,60)$ & $15(48,40)$ & & \\
\hline \multicolumn{5}{|l|}{ Tempo cirúrgico (horas) } \\
\hline Até 3 & $101(85,60)$ & $17(14,40)$ & \multirow{2}{*}{$0,87(0,45-1,7)$} & \multirow{2}{*}{0,695} \\
\hline Mais de 3 & $66(83,50)$ & $13(16,40)$ & & \\
\hline \multicolumn{5}{|l|}{ Inserção de drenos } \\
\hline Com drenos & $24(68,50)$ & $11(31,40)$ & \multirow{2}{*}{$4,48(2,39-8,41)$} & \multirow{2}{*}{$<0,001$} \\
\hline Sem drenos & $143(92,20)$ & $12(7,70)$ & & \\
\hline \multicolumn{5}{|l|}{ Internação na UTI } \\
\hline Com internação & $23(76,60)$ & $7(23,30)$ & \multirow{2}{*}{$1,91(0,73-4,94)$} & \multirow{2}{*}{0,181} \\
\hline Sem internação & $144(86,20)$ & $23(13,70)$ & & \\
\hline \multicolumn{5}{|c|}{ Pós-operatório na enfermaria (dias) } \\
\hline Até 3 & $108(93,10)$ & $8(6,90)$ & $503(211-1201)$ & $<0 \cap 01$ \\
\hline Mais de 3 & $59(72,80)$ & $22(27,10)$ & $0,03(2,11-\mid 2,01)$ & $<0,001$ \\
\hline
\end{tabular}

OR: odds ratio; IC95\%: índice de confiança de 95\%; UTI: unidade de terapia intensiva. 
que aumentam o risco, mas a idade de forma isolada não constitui fator de risco significativo.

Em relação ao risco aumentado de complicações pós-operatórias e IMC, há controvérsias, uma vez que, no presente estudo, apesar de haver maior número de pacientes com obesidade grau III e superobesidade, não foi possível realizar uma associação que evidenciasse maior incidência de complicações nesse grupo, como ocorreu em outra pesquisa nacional ${ }^{14}$.

As opções de cirurgia para tratamento da obesidade incluem o bypass em Y de Roux, gastrectomia vertical ou sleeve, banda gástrica e derivação biliopancreática. $O$ bypass é o procedimento mais realizado no Brasil e no mundo e, apesar de amplamente realizado, não é isento de complicações ${ }^{14,15}$. Optou-se por analisar as cirurgias de bypass e sleeve por serem os procedimentos mais utilizados na instituição para tratamento da obesidade. No período estudado, a realização de ambos procedimentos foi semelhante, com 51,3\% de bypass e $48,7 \%$ de sleeve. Além disso, com os avanços da cirurgia minimamente invasiva e o desenvolvimento da técnica videolaparoscópica, a cirurgia por vídeo passou a ser a mais utilizada mundialmente ${ }^{5,16}$. Dessa maneira, constata-se que foram realizadas, na presente amostra, mais cirurgias videolaparoscópicas em comparação com as convencionais / abertas ( 84,3 versus $15,7 \%$ ). Apesar de requerer maior curva de aprendizagem e desenvolvimento de habilidades laparoscópicas avançadas, a técnica por vídeo apresenta significativamente menores índices de complicações e mortalidade ${ }^{17}$.

O aumento do acesso a essas cirurgias repercute em aumento dos custos com o tratamento da obesidade; contudo, o custo em relação aos benefícios para os pacientes as torna viáveis e, considerando-se as complicações da doença, pode ser até menor ${ }^{18-}$ ${ }^{20}$. Os custos diminuem com o tempo e aumentam diretamente quando ocorrem complicações cirúrgicas ${ }^{20}$. Quanto mais as cirurgias com indicação demoram a ser realizadas, maiores são os custos e os riscos pré-operatórios ${ }^{21}$. Nesse segmento, o estudo mostra uma elevada ocorrência de complicações pós-operatórias de sítio cirúrgico na técnica bypass em Y de Roux com 19,8\% (OR=1,90) e taxa de 48,4\% (OR=5,35) de complicações na abordagem convencional/aberta.

Uma revisão sistemática encontrou taxas de reoperação de 6,7 a $24,0 \%$ para o bypass em $Y$ de Roux por videolaparoscopia; para o sleeve gástrico, de 3,3 a 34,0\% ${ }^{22}$. Efeitos nas comorbidades, complicações e procedimentos cirúrgicos adicionais não foram diferentes nos estudos avaliados, exceto em um ensaio clínico randomizado que encontrou mais refluxo gastroesofágico entre os pacientes submetidos ao bypass ${ }^{22}$. Ambos os procedimentos apresentaram melhores resultados do que a banda gástrica; contudo, a técnica associada à melhor perda de peso foi a derivação biliopancreática ${ }^{22}$. Já em outra revisão, a bandagem gástrica foi referida como a técnica que menos reduz o peso corporal e que resulta em menos reoperações e menos complicações ${ }^{23}$. Os autores relatam, por fim, que as complicações são pobremente descritas nos estudos, o que é relatado da mesma forma em outras revisões sistemáticas, o que justifica a dificuldade de se encontrar resultados que corroborem os achados desta pesquisa ou que sejam divergentes ${ }^{22,23}$. A perda de peso descrita na literatura é similar entre as duas técnicas estudadas ${ }^{22,23}$.

Drenos estavam presentes em $17,8 \%$ dos pacientes operados, e seu uso foi associado à maior chance de complicações pós-operatórias de sítio cirúrgico $(\mathrm{OR}=4,48)$. A finalidade da colocação ou não do dreno não pôde ser avaliada; porém, entende-se que a manutenção do dreno de sucção por longos períodos não evita o surgimento de complicações pós-operatórias, incluindo-se as ISC ${ }^{2}$.

Um estudo retrospectivo com 408 casos de cirurgias de bypass em Y de Roux e sleeve gástrico, apenas por técnica videolaparoscópica, evidenciou que as complicações acometeram 7,3\% da amostra, com incremento significativo nos riscos para maiores IMC pré-operatórios e no dia da cirurgia, para maior tempo cirúrgico e menor número de grampos utilizados ${ }^{16}$. O tipo de cirurgia, no estudo citado, não apresentou diferença significativa quanto à incidência de complicações perioperatórias ${ }^{16}$.

Para prevenção de complicações, o hospital deve contar com protocolo para profilaxia tromboembólica, incluindo botas pneumáticas e meias compressivas adequadas, além de terapia farmacológicaa $^{2425}$. As complicações mais comuns são: embolismo de artéria pulmonar, sangramento e fístula de anastomose e úlcera marginal, que requer uso de protetores gástricos por seis meses ${ }^{16}$.

Foi desenvolvido um índice com valor preditivo de complicações pós-operatórias da cirurgia bariátrica, o Bariatric Surgery Index for Complications (BASIC), ainda por ser traduzido e validado para o português ${ }^{26}$. Por esse índice, os pacientes são classificados em: classe I, quando apresentam de zero a um fator de risco; classe II, com dois fatores; e classe III, com três ou mais fatores de risco ${ }^{26}$. No estudo de elaboração do índice, as complicações variaram por classe de forma significativa, entre 13,$5 ; 21,6$ e $31,4 \%$, respectivamente ${ }^{26}$.

A atuação no cuidado perioperatório específica é fundamental e tem como objetivo prevenir e auxiliar no tratamento de possíveis complicações, além de oferecer apoio, iniciando antes da cirurgia bariátrica e se estendendo no pós-operatório. Como membro da equipe multidisciplinar, o enfermeiro deve assegurar cuidados e educação em saúde, auxiliando 
o paciente na sua adaptação à nova maneira de viver. Um estudo recente evidenciou que pacientes que passaram por um processo de educação pré-operatória tiveram alta em menos tempo que o grupo controle, em média no primeiro dia após a cirurgia, contra dois dias para o grupo que não recebeu orientação, o que ressalta o papel do enfermeiro no processo e suas possibilidades de atuação ${ }^{27}$.

Por fim, o presente estudo apresentou como limitações: o tipo de coleta (dados secundários); o tamanho da amostra em relação à prevalência dos eventos, o que dificultou outras associações significativas entre as variáveis; e o fato de ter sido realizado em um único hospital. Não foi testada a associação entre o tipo de dreno (por sucção ou drenagem ativa) com a presença de infecções ou de seroma. Além disso, os pacientes não foram estratificados em grupos de risco de complicações antes de serem incluídos no estudo.

\section{CONCLUSÃO}

Entre os 197 pacientes submetidos a cirurgias bariátricas que compuseram a amostra, 30 apresentaram 45 complicações. Como predisponentes a um maior desenvolvimento de complicações, comprovou-se: maior prevalência de seroma como complicação de sítio cirúrgico, técnica cirúrgica bypass em Y de Roux, faixa etária maior de 45 anos, tipo de abordagem cirúrgica convencional/aberta, presença de inserção de dreno cavitário e tempo de internação superior a 3 dias.

Dentre as principais contribuições dessa pesquisa, está o feedback dado ao serviço de saúde onde o estudo foi realizado, para elaboração de planejamento de ações preventivas, por meio da descrição dos principais fatores de risco associados às complicações de ferida operatória após cirurgias bariátricas.

O presente estudo demonstrou a importância de uma avaliação adequada desses pacientes, sendo indispensável a análise de todos os critérios que possam influenciar no surgimento de complicações pós-operatórias de sítio cirúrgico - que acarretam problemas emocionais, maior tempo de internação, custos elevados ao serviço, além de complicações severas (que podem ocasionar elevado número de óbitos nessa população).

Dessa maneira, a equipe de enfermagem precisa estar sempre atenta e auxiliar na identificação precoce, além da realização de cuidados adequados para a prevenção e o tratamento desses tipos de complicação.

Este estudo serve para preencher lacunas a respeito dessa temática, como também para incentivar gestores da saúde a disponibilizarem recursos ao desenvolvimento de mais pesquisas sobre essa problemática. Sugere-se que pesquisas posteriores continuem a investigação, avaliando outros fatores, como a associação entre o tipo de dreno e o tipo de infecção, e viabilizem a elaboração e validação da sistematização da assistência de enfermagem para pacientes com complicações advindas da cirurgia bariátrica.

\section{REFERÊNCIAS}

1. Fry BT, Scally CP, Thumma JR, Dimick JB. Quality improvement in bariatric surgery: the impact of reducing postoperative complications on medicare payments. Ann Surg. 2017 Dec 4. doi: 10.1097/SLA.0000000000002613

2. Doble B, Wordsworth S, Rogers CA, Welbourn R, Byrne J, Blazeby $J M$, et al. What are the real procedural costs of bariatric surgery? A systematic literature review of published cost analyses. Obes Surg. 2017;27(8):2179-92. DOI: 10.1007/s11695-017-2749-8

3. Brasil. Ministério da Saúde (MS). Portaria n. 425, de 19 de março de 2013. Redefine as diretrizes para a organização da prevenção e do tratamento do sobrepeso e obesidade como linha de cuidado prioritária da Rede de Atenção à Saúde das Pessoas com Doenças Crônicas. Brasília: Ministério da Saúde; 2013.

4. Straciere LDS. Cuidados e complicações pós-operatórias. Medicina. 2008;41(4):465-8.

5. Nguyen NT, Goldman C, Rosenquist CJ, Arango A, Cole CJ, Lee SJ, et al. Laparoscopic versus open gastric bypass: a randomized study of outcomes, quality of life, and costs. Ann Surg. 2001;234(3):279-89.
6. Encinosa WE, Bernard DM, Du D, Steiner CA. Recent improvements in bariatric surgery outcomes. Med Care. 2009;47(5):531-5. DOI: 10.1097/MLR.0b013e31819434c6

7. Périssé LGS, Périssé PCM, Bernardo Júnior C. Tratamento endoscópico das fístulas após gastrectomia vertical e by-pass gástrico em Y de Roux. Rev Col Bras Cir. 2015;42(3):159-64. DOI: 10.1590/0100-69912015003006

8. Carvalho DV, Borges EL. Tratamento ambulatorial de pacientes com ferida cirúrgica abdominal e pélvica. Rev Min Enferm. 2011;15(1):25-33.

9. Chang SH, Stoll CRT, Song JJ, Varela E, Eagon CJ, Colditz GA. The effectiveness and risks of bariatric surgery an updated systematic review and meta-analysis, 2003-2012. JAMA Surg. 2014;149(3):27587. DOI: 10.1001/jamasurg.2013.3654

10. Pajecki D, Santo MA, Joaquim HDG, Morita F, Riccioppo D, Cleva $\mathrm{R}$, et al. Cirurgia bariátrica em idosos: resultados de seguimento de cinco anos. ABCD Arq Bras Cir Dig. 2015;28(Supl. 1):15-8. DOI: /10.1590/S0102-6720201500S100006 
11. Brasil. Ministério da Saúde (MS). Portaria n. 390 -SAS/MS (Secretaria de Atenção à Saúde/ Ministério da Saúde), de 06 de julho de 2005. Unidade de assistência em alta complexidade ao paciente portador de obesidade grave. Brasília: Ministério da Saúde; 2005.

12. Conselho Federal de Medicina (CFM). Resolução n. 1.942, de 12 de fevereiro de 2010. Altera a Resolução CFM n. 1.766, de 13 de maio de 2005, publicada no Diário Oficial da União em 11 de julho de 2005, Seção I, página 114. Estabelece normas seguras para o tratamento cirúrgico da obesidade mórbida, definindo indicações, procedimentos e equipe. Brasília: Conselho Federal de Medicina; 2010.

13. Abbas M, Cumella L, Zhang Y, Choi J, Vemulapalli P, Melvin WS, et al. Outcomes of laparoscopic sleeve gastrectomy and roux-en-y gastric bypass in patients older than 60. Obes Surg. 2015;25(12):2251-6. DOI: $10.1007 / \mathrm{s} 11695-015-1712-9$

14. Wrzesinski A, Corrêa JM, Fernandes TMB, Monteiro, LF, Trevisol, FS, Nascimento RR. Complicações que necessitaram de manejo hospitalar no pós-operatório de cirurgia bariátrica. ABCD Arq Bras Cir Dig. 2015;28(Supl. 1):3-6. DOI: /10.1590/ S0102-6720201500S100003

15. Eldar S, Heneghan HM, Brethauer SA, Schauer PR. Bariatric surgery for treatment of obesity. Int J Obes. 2011;35(Supl. 3):S16-21. DOI: 10.1038/ijo.2011.142

16. Major P, Wysocki M, Pędziwiatr M, Pisarska M, Dworak J, Matczak P, et al. Risk factors for complications of laparoscopic sleeve gastrectomy and laparoscopic Roux-en-Y gastric bypass. Int J Surg. 2017;37:718. DOI: 10.1016/j.ijsu.2016.12.012

17. Rausa E, Bonavina L, Asti E, Gaeta M, Ricci C. Rate of death and complications in laparoscopic and open Roux-en-y gastric bypass: a meta-analysis and meta-regression analysis on 69,494 patients. Obes Surg. 2016;26(8):1956-63. DOI: 10.1007/s11695-016-2231-z

18. Kamei DJ, Sampaio Neto J, Nassif LS, Balbino IB, Broska AC, Nassif AT. Análise da obstrução intestinal relacionada à cirurgia aberta de bypass gástrico. ACM Arq Catarin Med. 2016;45(4):78-83.
19. Kelles SMB, Diniz MFHS, Machado CJ, Barreto SM. Perfil de pacientes submetidos à cirurgia bariátrica, assistido pelo Sistema Único de Saúde do Brasil: revisão sistemática. Cad Saúde Pública. 2015;31(8):1587601. DOI: http://dx.doi.org/10.1590/0102-311X00022714

20. Gulliford MC, Charlton J, Prevost T, Booth H, Fildes A, Ashworth M, et al. Costs and outcomes of increasing access to bariatric surgery: cohort study and cost-effectiveness analysis using electronic health records. Value Health. 2017;20(1):85-92. DOI: 10.1016/j.jval.2016.08.734

21. Cohen RV, Luque A, Junqueira S, Ribeiro RA, Le Roux CW. What is the impact on the healthcare system if access to bariatric surgery is delayed? Surg Obes Relat Dis. 2017;13(9):1619-27. DOl: 10.1016/j.soard.2017.03.025

22. Colquitt JL, Pickett K, Loveman E, Frampton GK. Surgery for weight loss in adults. Cochrane Database Syst Rev. 2014;(8):CD003641. DOI: $10.1002 / 14651858$

23. Padwal R, KlarenbachS, Wiebe N, HazelM, BirchD, KarmaliS, et al. Bariatric surgery: a systematic review of the clinical and economic evidence. J Gen Intern Med. 2011;26(10):1183-94. DOI: 10.1007/s11606-011-1721-x

24. Cammarota MC, Ribeiro Júnior I, Lima RQ, Almeida CM, Moura LG, Daher LMC, et al. Estudo do uso de pontos de adesão para minimizar a formação de seroma após mastectomia com reconstrução imediata. Rev Bras Cir Plást. 2016;31(2):158-65. DOI: http://www.dx.doi. org/10.5935/2177-1235.2016RBCP0026

25. Wu H, Zhong M, Zhou D, Shi C, Jiao H, Wu W, et al. Prevention, diagnosis and treatment of perioperative complications of bariatric and metabolic surgery. Zhonghua Wei Chang Wai Ke Za Zhi. 2017;20(4):393-7.

26. Coblijn UK, Karres J, de Raaff CAL, de Castro SMM, Lagarde SM, Van Tets WF, et al. Predicting postoperative complications after bariatric surgery: the Bariatric Surgery Index for Complications, BASIC. Surg Endosc. 2017;31(11):4438-45. DOI: 10.1007/s00464-017-5494-0

27. Sheaffer WW, Day RW, Harold KL, Kidwell JT, Tiede JL, Benjamin TR, et al. Decreasing length of stay in bariatric surgery: the power of suggestion. Am J Surg. 2017. DOI: http://dx.doi.org/10.1016/j.amjsurg.2017.09.041 